\title{
The Identification Nanoparticle Sensor Using Back Propagation Neural Network Optimized by Genetic Algorithm
}

\author{
Yiwen Hu $\mathbb{D},,^{1}$ Ashutosh Sharma $\mathbb{D}^{2}$, Gaurav Dhiman $\mathbb{D}^{3}$ and Mohammad Shabaz $\mathbb{D}^{4}$ \\ ${ }^{1}$ Testing Center of Aviation Theory, Civil Aviation Flight University of China Guanghan, Sichuan 618307, China \\ ${ }^{2}$ Institute of Computer Technology and Information Security, Southern Federal University, Russia \\ ${ }^{3}$ Government Bikram College of Commerce, Punjab, India \\ ${ }^{4}$ Arba Minch University, Ethiopia \\ Correspondence should be addressed to Yiwen Hu; yiwenhu21@outlook.com \\ and Mohammad Shabaz; mohammad.shabaz@amu.edu.et
}

Received 9 June 2021; Revised 6 October 2021; Accepted 1 November 2021; Published 18 November 2021

Academic Editor: Alfian Abdul Halin

Copyright (c) 2021 Yiwen Hu et al. This is an open access article distributed under the Creative Commons Attribution License, which permits unrestricted use, distribution, and reproduction in any medium, provided the original work is properly cited.

\begin{abstract}
This study draws attention towards the application of identification nanoparticle (NPs) sensor based on back propagation (BP) neural network optimized by genetic algorithm (GA) in the early diagnosis of cancer cells. In this study, the traditional and optimized BP neural networks are compared in terms of error between the actual value and the predictive value, and they are further applied to the NP sensor for early diagnosis of cancer cells. The results show that the root mean square (RMS) and mean absolute error (MAE) of the optimized BP neural network are comparatively much smaller than the traditional ones. The particle size of silicon-coated fluorescent NPs is about $105 \mathrm{~nm}$, and the relative fluorescence intensity of silicon-coated fluorescent NPs decreases slightly, maintaining the accuracy value above $80 \%$. In the fluorescence imaging, it is found that there is obvious green fluorescence on the surface of the cancer cells, and the cancer cells still emit bright green fluorescence under the dark-field conditions. In this study, a phenolic resin polymer CMK-2 with a large surface area is successfully combined with Au. NPs with good dielectric property and bioaffinity are selectively bonded to the modified electrode through a sulfur-gold bond to prepare NP sensor. The sensor shows good stability, selectivity, and anti-interference property, providing a new method for the detection of early cancer cells.
\end{abstract}

\section{Introduction}

In the past few years, people's increasingly high requirements for microscopic detection technology have led to an evolution, in the applications of various nanomaterials in the biological field. NPs refer to particles with a particle size between 1 and $100 \mathrm{~nm}$, and it is a new material for research at the molecular level, cellular level, and subcellular level [1]. The NP-based nanotechnology can be applied to the diagnosis and treatment of tumors [2].

Fluorophores can be added to NPs by covalent bonding or embedding to form fluorescent NPs [3], which are used as fluorescent dyes for detection or labeling. Compared with ordinary fluorescent dyes, fluorescent NPs have high brightness, good stability, strong water solubility, and good biocompatibility [4]. Cancer cell imaging is of great significance in the diagnosis of tumors. As a carrier material, fluorescent NPs can enhance biological signals to facilitate detection [5]. Hyaluronic acid (HA) has a good application prospect in the diagnosis and treatment of tumor diseases [6]. A nanodrug-carrying system prepared by combining NPs and HA can specifically bind to receptor proteins on the cell surface to detect cancer cells. Shen et al. [7] prepared diamine-hyaluronic acid-iron-modified rhodamine fluorescent NPs for cancer imaging detection. The results proved that fluorescent NPs had high sensitivity and stability.

Sensors play an important role in various fields, and chemical sensors are generally used to detect chemical substances. Electrochemical biosensors use active substances in organisms as sensitive elements. It converts them into electrochemical signals to output characteristic signals such as potential, current, and capacitance [8]. The principle of 


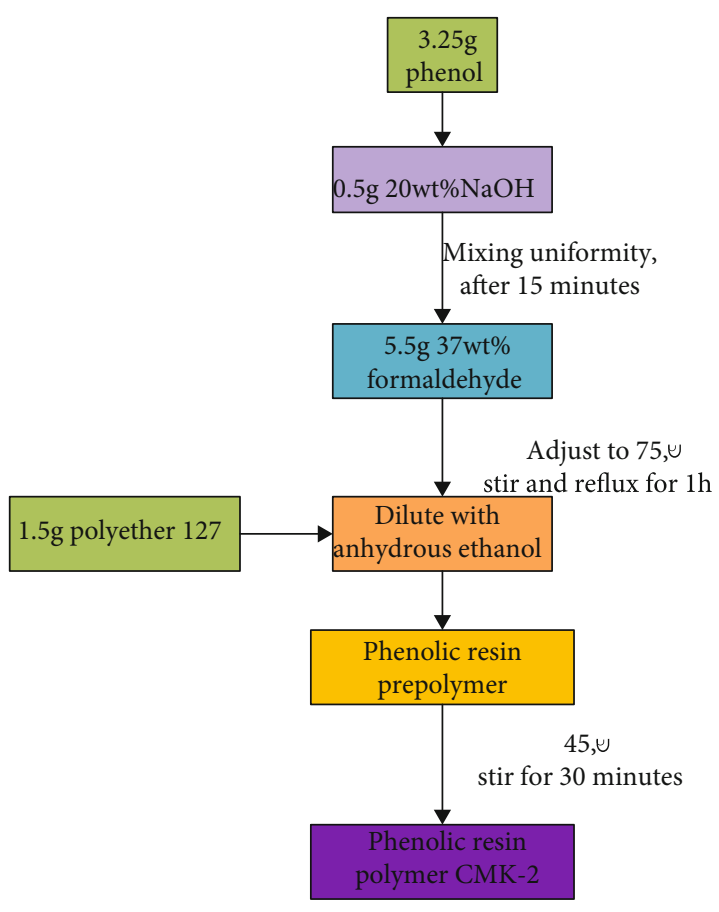

Figure 1: Preparation of CMK-2.

electrochemical biosensor is as follows. When the active substance in the organism diffuses to the biosensitive layer, relevant chemical reactions occur. The biological signals are then converted into electrical signals that can be quantitatively analyzed. Finally, the concentration of the active substance can be identified by processing the electrical signals [9]. Nanomaterials have many functions in electrochemical sensors. They can modify electrodes, accelerate the rate of electron transfer, promote catalytic reactions, and immobilize biomolecules. They can further be used as carriers for markers, control switches for chemical reactions, as well as reactants [10]. The introduction of NPs into electrochemical biosensors can effectively improve its sensitivity and reproducibility.

The genetic algorithm (GA) is a probabilistic adaptive iterative algorithm that can process the discontinuous function through the global search [11], as it finds the global optimal solution by not being affected by gradient information. It can be used to optimize BP network [12]. When the optimized BP neural network based on GA is applied to the NP sensor, it endows the nanoparticle sensor with ideal stability by adjusting the weight and threshold [13].

In the past studies depicted in this section, it is revealed that alone neural network-based approaches do not provides satisfactory outcomes for finding optimal global solution for diagnosis of cancer cells and this is a great research challenge. In the study, the GA is used to optimize the BP neural network. The fluorescent NPs are applied in electrochemical sensors, with their basic characteristics expounded. The modified electrodes of Me- $\beta-\mathrm{Cy} /$ $\mathrm{Au}$ NPs/CMK-2/GCE are introduced in the biosensor which is applied in the early diagnosis of cancer cells, aimed at exploring the diagnostic value of fluorescent NP sensors for cancer cells.

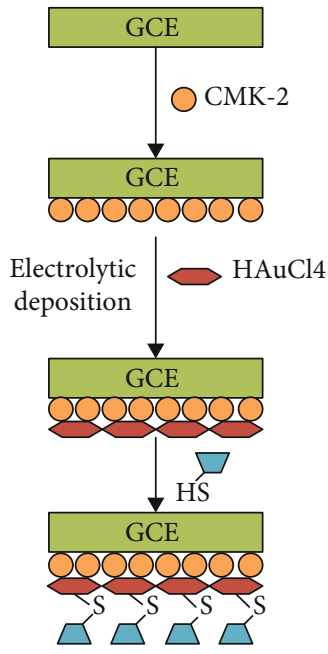

Figure 2: The preparation of $\mathrm{Me}-\beta-\mathrm{Cy} / \mathrm{Au} \mathrm{NPs} / \mathrm{CMK}-2 / \mathrm{GCE}$.

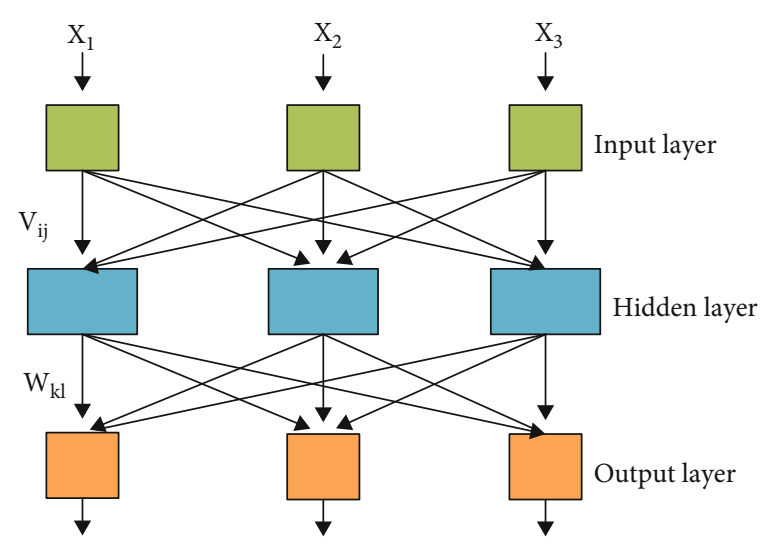

FIgURE 3: The BP neural network.

\section{Experimental Methodology}

\subsection{Me- $\beta$-Cy/Au NPs/CMK-2/GCE Electrodes}

\subsubsection{Preparation of $\mathrm{CMK}-2$}

(1) Preparation of the Phenolic Resin Prepolymer. $3.25 \mathrm{~g}$ of phenol is dissolved in $0.5 \mathrm{~g}$ of $20 \mathrm{wt} \% \mathrm{NaOH}$ solution. 15 minutes later, the uniform mixture is slowly dripped into $5.5 \mathrm{~g}$ of $37 \mathrm{wt} \%$ formaldehyde solution, with the temperature adjusted to $75^{\circ} \mathrm{C}$, followed by reflux for $1 \mathrm{~h}$. After the mixture cools to $25^{\circ} \mathrm{C}, 0.4 \mathrm{~mol} / \mathrm{L} \mathrm{HCl}$ is used to adjust $\mathrm{pH}=7$, followed by evaporation treatment. The mixture is then diluted with absolute ethanol. After the white particles of $\mathrm{NaCl}$ are removed by filtering, the remaining light-yellow solution is the phenolic resin prepolymer.

(2) Preparation of CMK-2. $1.5 \mathrm{~g}$ of polyether F127 is added to the solution prepared above, with the temperature adjusted to $45^{\circ} \mathrm{C}$, followed by stirring for $30 \mathrm{~min}$ to form a lightyellow transparent solution. The mixture is then transferred to a petri dish at $45^{\circ} \mathrm{C}$ and dried for 8 hours to remove ethanol. The temperature is adjusted again to $100^{\circ} \mathrm{C}$ for thermal polymerization for 24 hours to form the transparent film, 


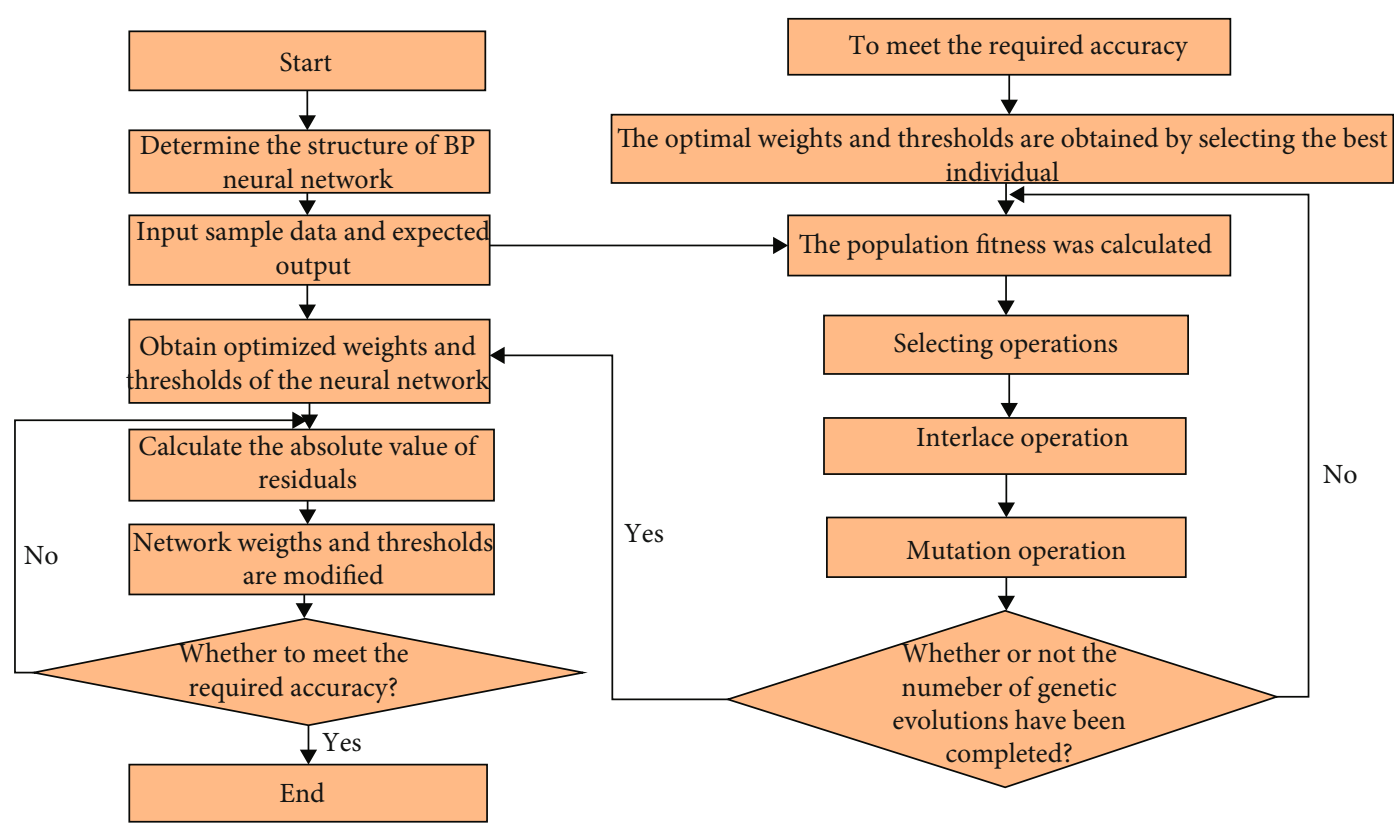

FIgURE 4: The optimization of the BP neural network by GA.

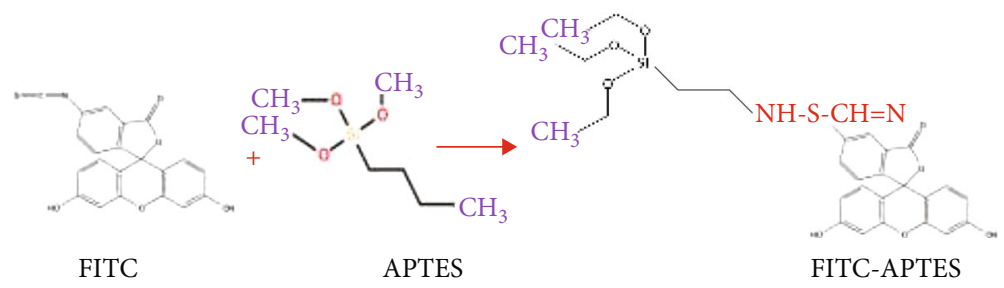

FIgURE 5: The preparation of FITC-APTES.

which is pulverized and fired at $350^{\circ} \mathrm{C}$ for 5 hours under nitrogen conditions to finally obtain dark brown powder. The powder is then dispersed in $98 \%$ concentrated sulfuric acid solution, followed by reflux for 24 hours. After the template polyether F127 is removed, CMK-2 is finally obtained, as shown in Figure 1.

2.1.2. Preparation of Modified Electrode. The glassy carbon electrode (GCE) is polished with $0.2 \mu \mathrm{m}$ and $0.05 \mu \mathrm{m}$ alumina wet powder, respectively, and then undergoes ultrasonic cleaning in absolute ethanol and ultrapure water, respectively. The control volume $(\mathrm{CV})$ method is used for scanning activation in a $5 \mathrm{mmol} / \mathrm{L}$ sulfuric acid solution [14], with potential controlled at $-0.5-1.6 \mathrm{~V}$, and the scanning rate set as $100 \mathrm{mV} / \mathrm{s}$. The electrode is then cleaned with ultrapure water and dried at room temperature.

$1.8 \mathrm{mg}$ of CMK-2 powder prepared above is dissolve in $1 \mathrm{~mL}$ of dimethylformamide (DMF), followed by ultrasound treatment for $1 \mathrm{~h}$ to form a uniformly dispersed black suspension. Subsequently, $2.5 \mu \mathrm{L}$ of the suspension is applied to bare GCE, dried at room temperature, and recorded as a CMK-2/GCE electrode [15]. The above electrode is placed in $0.6 \mathrm{mmol} / \mathrm{L}$ chloroauric acid $\left(\mathrm{HAuCl}_{4}\right)$ (Sigma-Aldrich, USA) solution for electrodepositing gold (Au NPs), with potential controlled at $-0.5-1.6 \mathrm{~V}$. The $\mathrm{CV}$ method is used

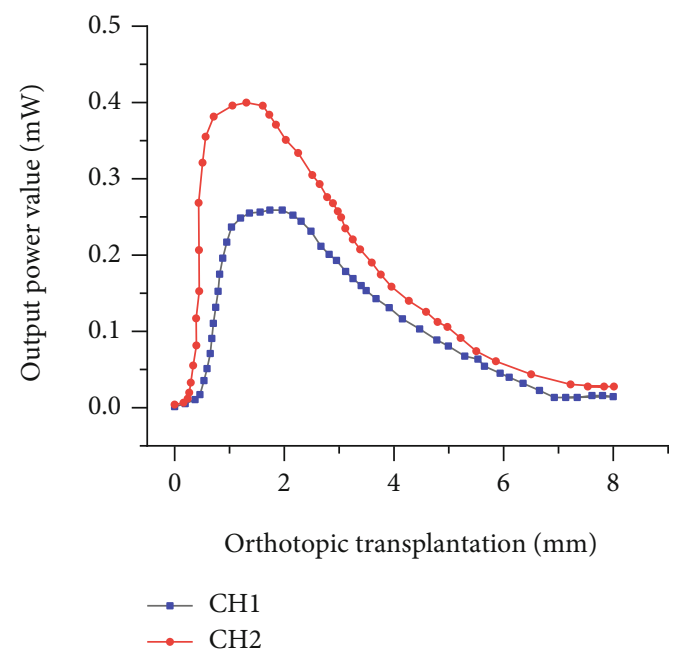

FIGURE 6: The relationship between displacement and received power.

to scan 10 times, with the scanning speed set as $50 \mathrm{mV} / \mathrm{s}$, followed by rinse step using ultrapure water. It is then dried at room temperature and recorded as Au NPs/CMK-2/GCE. The above electrode is immersed in a phosphate buffer solution containing $1 \mathrm{mmol} / \mathrm{L} \mathrm{Me}-\beta-\mathrm{Cy}$ (Binzhou Zhiyuan 


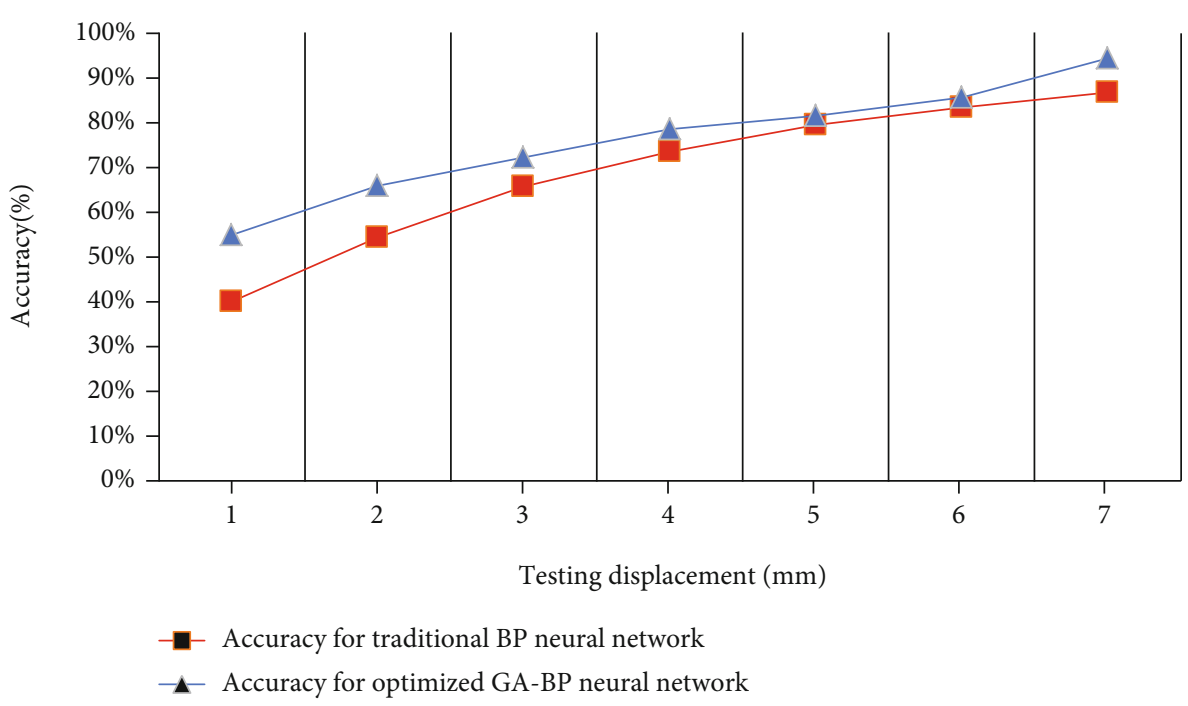

FIGURE 7: Accuracy values for traditional and optimized GA-BP neural networks.

Biotechnology, China) for assembling for $12 \mathrm{~h}$, followed by rinse with ultrapure water. It is then dried at room temperature [16], denoted as Me- $\beta$-Cy/Au NPs/CMK-2/GCE, as shown in Figure 2.

2.2. The BP Neural Network Improved by GA. The BP neural network is a kind of feedforward neural network [17]. It is generally composed of an input layer, a hidden layer, and an output layer (Figure 3). The BP neural network has good self-learning and self-adaptation capabilities and strong fault tolerance [18].

In the study, the GA is used to optimize the initial weight threshold of the BP neural network, with the general initial weight search replaced by global search, so that the BP algorithm can accurately debug the neural network in the solution space, and search for the global optimal solution [19]. The optimization includes population initialization, fitness function selection, selection operation, crossover operation, and mutation operation [20], as shown in Figure 4.

After the training of the BP neural network, the sum of the residual absolute value between the predicted output and the expected output is set as the fitness function $H$.

$$
H=l\left(\sum_{j=1}^{m}\left|y_{j}-p_{j}\right|\right) \text {, where } y_{j} \neq p_{j} \text {, }
$$

where $l$ represents the coefficient, and it is 1 in the study. $m$ represents the value of the output node, $y_{j}$ represents the expected output value of the $j$ point, and $p_{j}$ represents the predicted output value of the $j$ point. The probability of each individual is expressed as follows:

$$
P_{j}=H \sum_{j=1}^{M} H_{j}
$$

where $H$ represents the fitness function and $P_{j}$ represents the probability of an individual $j$. In this study, the real number

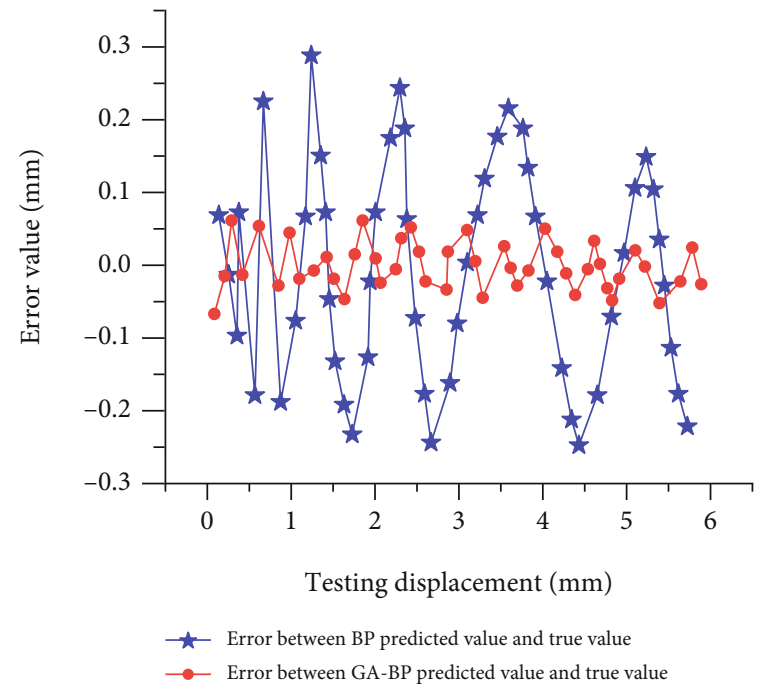

Figure 8: Comparison of traditional and optimized BP neural networks.

crossover method is applied, and the crossover operation equation of the $l$ chromosome $g_{1}$ and the $k$ chromosome $g_{k}$ at the point $i$ is as follows:

$$
\begin{gathered}
g_{l i}=g_{l i}(1-a)+g_{k i} a, \\
g_{k i}=g_{k i}(1-a)+g_{l i} a,
\end{gathered}
$$

where $a$ is within $[0,1]$. If the $i$ gene of the $j$ individual is selected for mutation processing, the operating equation is as follows:

$$
g_{i j}= \begin{cases}g_{i j}+\left(g_{i j}-g_{\max }\right) Q(f), & \lambda>0.5, \\ g_{i j}+\left(g_{\min }-g_{i j}\right) Q(f), & \lambda \leq 0.5,\end{cases}
$$




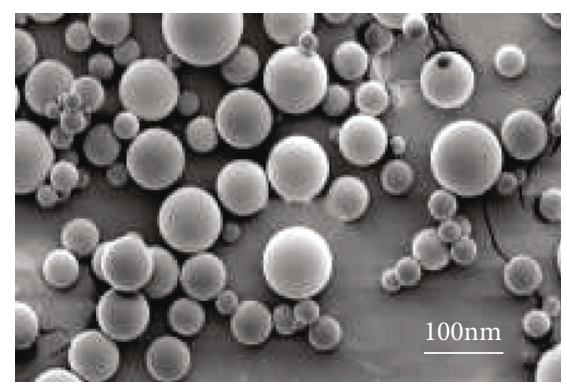

(a)

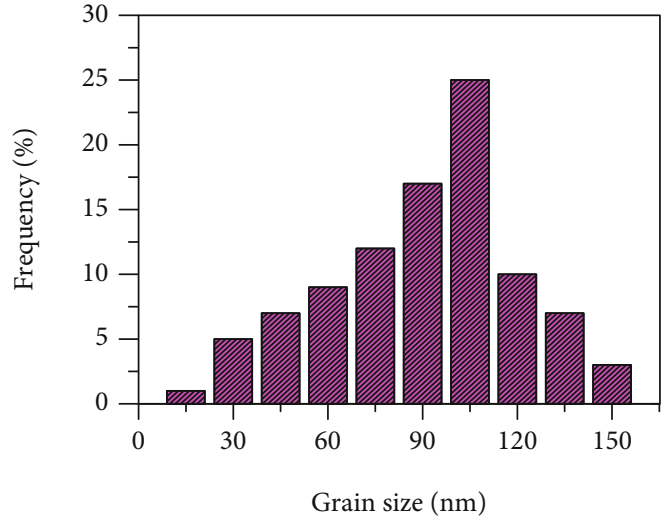

(b)

FIGURE 9: Morphology and particle size of silicon-coated fluorescent NPs. Note: (a) electron micrograph and (b) particle size distribution.

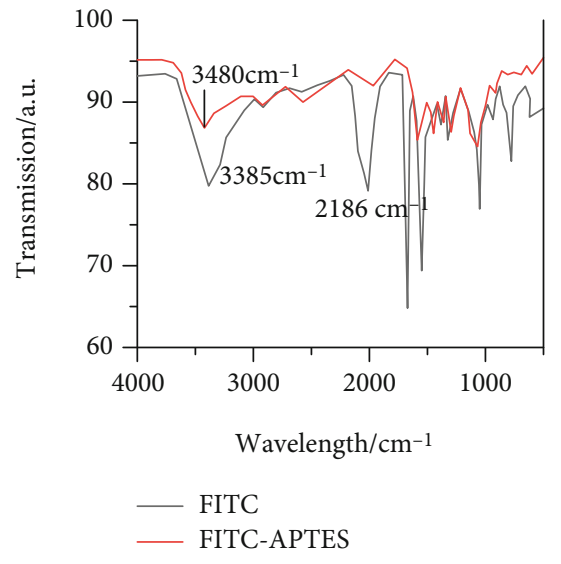

(a)

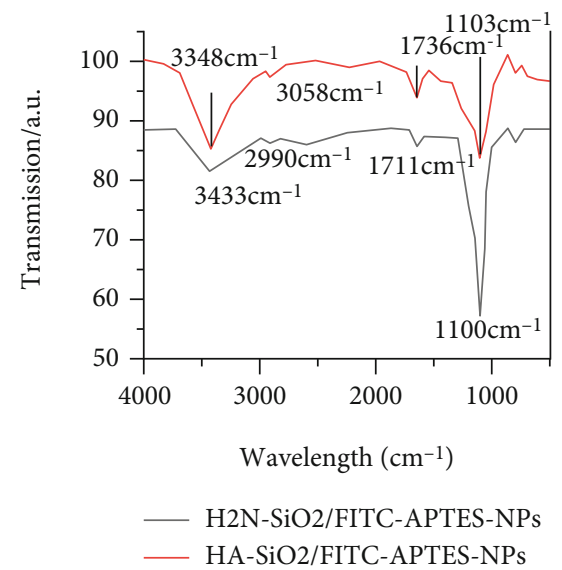

(b)

FIGURE 10: Infrared spectrum of modified electrode. Note: (a) infrared spectra of FITC and FITC-APTES precursors and (b) infrared spectra of $\mathrm{H}_{2} \mathrm{~N}-\mathrm{SiO}_{2}$ /FITC-APTES-NPs and $\mathrm{HA}-\mathrm{SiO}_{2} /$ FITC-APTES-NPs.

$$
Q(f)=\lambda^{\prime}\left(\frac{1-f}{F_{\max }}\right)^{2},
$$

where $g_{\max }$ is the maximum of $g_{i j}, g_{i j}$ is the minimum, $f$ is the number of iterations, $F_{\max }$ is the maximum number of evolutions, and $\lambda$ and $\lambda^{\prime}$ are within $[0,1]$.

The best optimized weight values are obtained by using GA methodology, and the weight and threshold values are optimized. It is further followed by the calculation of absolute values of residual, and then, the network weights are further modified. The accuracy requirements are then checked, and the entire optimization process of the BP neural network is then terminated.

\subsection{The Fluorescent NPs Modified by HA}

2.3.1. The Silicon-Coated Fluorescent NPs. $1.5 \mathrm{mg}$ of fluorescein isothiocyanate (FITC) (Sigma-Aldrich, USA) is dissolved in $15 \mathrm{~mL}$ of double-distilled absolute ethanol, followed by ultrasound treatment. The solution is then dripped into anhydrous ethanol without distillation treat- ment, followed by magnetic stirring for 24 hours. Subsequently, the mixture is centrifuged and washed with anhydrous ethanol and n-hexanol alternately until there is no fluorescence in the supernatant. It is then dried in vacuum and stored in darkness [21]. $7.5 \mathrm{~mL}$ of cyclohexane, $2.5 \mathrm{~mL}$ of $\mathrm{n}$-hexanol, and $1.88 \mathrm{~mL}$ of Triton $\mathrm{x}-100$ are mixed uniformly, followed by magnetic stirring until it is clear. Subsequently, $250 \mu \mathrm{L}$ of ultrapure water is added, followed by stirring for $15 \mathrm{~min}$ to form a relatively stable microemulsion system [22].

Figure 5 shows the preparation process of FITC-APTES. $0.25 \mathrm{mg}$ of FITC and APTES (Sigma-Aldrich, USA) is dissolved in a mixture of $0.6 \mathrm{~mL}$ cyclohexane and $0.6 \mathrm{~mL} \mathrm{n}$-hexanol, followed by ultrasound treatment to fully dissolve them. The mixture is then dripped into the microemulsion system prepared above. After stirring for $30 \mathrm{~min}$, nucleation occurs. $125 \mu \mathrm{L}$ mixture of ethyl orthosilicate and 3aminopropyltriethoxysilane is added [23], together with $50 \mu \mathrm{L}$ ammonia water. After magnetic stirring for $24 \mathrm{~h}$, a few drops of acetone are added to demulsify. Subsequently, the sample is washed alternately with absolute ethanol and 


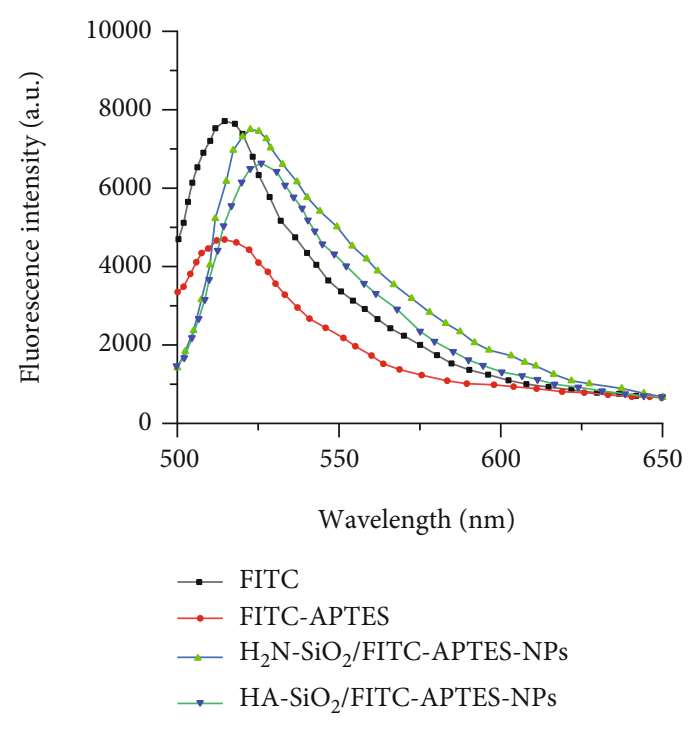

(a)

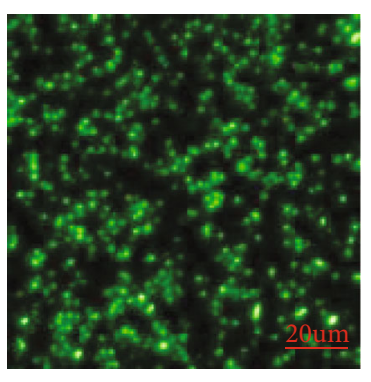

(b)

FIGURE 11: Fluorescence performance of silicon-coated fluorescent NPs. Note: (a) fluorescence spectra of FITC, FITC-APTES, $\mathrm{H}_{2} \mathrm{~N}-\mathrm{SiO}{ }_{2} /$ FITC-APTES-NPs, and HA-SiO 2 /FITC-APTES-NPs and (b) fluorescence confocal microscopy image of $\mathrm{HA}_{2} \mathrm{SiO}_{2} / \mathrm{FITC}$-APTES-NPs.

ultrapure water [24]. Finally, $\mathrm{H}_{2} \mathrm{~N}-\mathrm{SiO}_{2} /$ FITC-APTES-NPs are obtained and stored in refrigerator at $4^{\circ} \mathrm{C}$ for later use.

2.3.2. The HA Covalent Modification. $2.5 \mathrm{mg}$ of sodium HA $(\mathrm{NaA})$ is dissolved in $20 \mathrm{~mL}$ of ultrapure water by ultrasound treatment, with $0.02 \mathrm{mg}$ of carbodiimide (EDC) and $\mathrm{N}$ hydroxysuccinimide (NHS) (Ballingweier Reagent Company, China) added, respectively, followed by magnetic stirring for $2 \mathrm{~h}$ to activate the carboxyl group on HA [25]. $6 \mathrm{~mL}$ of the fluorescent NPs prepared above is then added, followed by stirring for $24 \mathrm{~h}$ under darkness. The ultrapure water is used for multiple washing. The unreacted HA and other small molecule impurities are removed through centrifugation [26]. Finally, HA covalently modified silicon-coated fluorescent NPs are obtained and stored at $4^{\circ} \mathrm{C}$ for later use.

\subsubsection{Characterization and Determination of Silicon-Coated} Fluorescent NPs. The electron microscope images of $\mathrm{HN}-$ $\mathrm{SiO}_{2} /$ FITC-APTES-NPs are measured using JEOL-2100 electron microscope (JEOL Company, Japan). A Nicolet Avatar 370 DTGS infrared spectrometer (Nicolet Avatar, USA) is used to obtain an infrared spectrum. The fluorescence spectrum is measured with Hitachi F-4600 fluorescence spectrophotometer (Hitachi, Japan) instrument. LSM 5 Pascal Laser Module laser scanning confocal microscope (Carl Zeiss, Germany) is used to characterize the results of cell incubation. A Bio-Rad Model 680 spectrophotometer (Bio-Rad Company, USA) is used to test the MTT results.

\subsection{Cultivation and Survival Rate of Cancer Cells}

2.4.1. Cultivation of Cancer Cells. Human cervical cancer Hela cells were cultured in DMEM containing 12\% fetal bovine serum, penicillin, and streptomycin at $37^{\circ} \mathrm{C}$ [27]. Hela cells in the proliferation phase were treated with trypsinization and seeded $\left(10^{4}-10^{5}\right.$ cells/well). $60 \mathrm{mg} / \mathrm{L} \mathrm{H}_{2} \mathrm{~N}-\mathrm{SiO}_{2} /$ FITC-APTES-NPs were then added to incubate the cells for

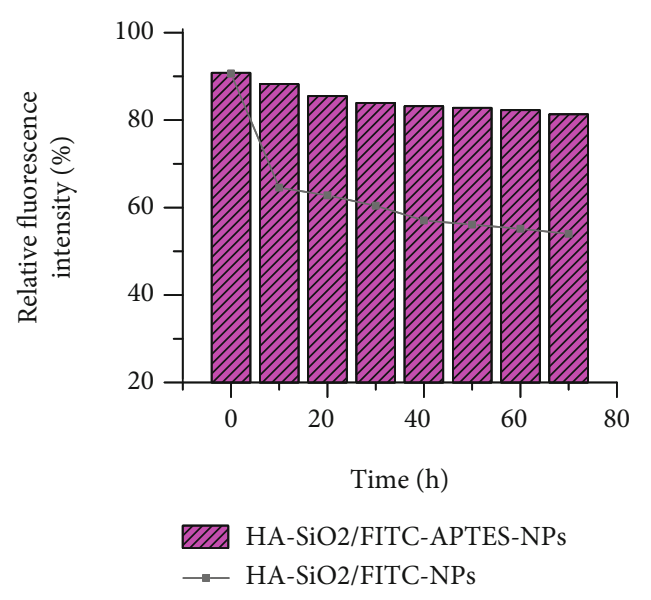

FIgURE 12: The dye leakage results.

4h. After the supernatant was removed, the sample was rinsed with phosphate buffer and visualized using LSM 5 Pascal Laser Module laser scanning microscope.

2.4.2. The Survival Rate Detection. The $\mathrm{H}_{2} \mathrm{~N}-\mathrm{SiO}_{2} /$ FITCAPTES-NPs were diluted using a phosphate buffer to configure solutions of $50,100,150,200,250$, and $300 \mathrm{mg} / \mathrm{mL}$, respectively. Hela cells were seeded at $10^{4}-10^{5}$ cells/well in a 96-well plate, and the abovementioned different concentrations of phosphate buffer is then transferred to the 96-well plate, followed by incubation at $37^{\circ} \mathrm{C}$ overnight [28]. After the medium is removed, $10 \mu \mathrm{L}$ of $1 \mathrm{mg} / \mathrm{mL}$ MTT (SigmaAldrich, USA) solution is added to each well to culture the cells for 4 hours, with the supernatant removed. $100 \mu \mathrm{L}$ sodium dodecyl sulfate (SDS) was then added to each well to culture the cells for 15 minutes, so that the MTT can fully 


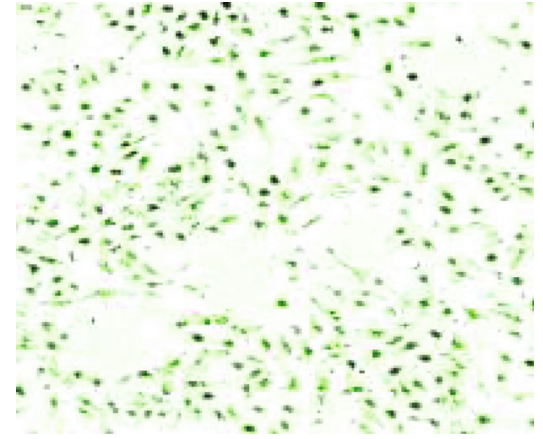

(a)

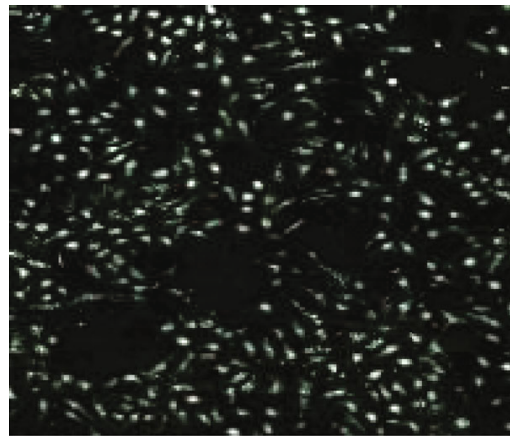

(b)

FIGURE 13: The fluorescence imaging of $\mathrm{HA}-\mathrm{SiO}_{2} /$ FITC-APTES-NPs. Note: (a) bright-field fluorescence imaging and (b) dark-field fluorescence imaging.

react with the cells. The Bio-Rad Model 680 microplate reader is used to measure the absorbance at $580 \mathrm{~nm}$.

2.5. Statistics. In this study, the data was processed by SPSS19.0, and all experimental data were expressed as mean \pm standard deviation $(\bar{x} \pm s)$. The root mean square (RMS) was applied to analyze the deviation between the predicted value and the expected value along with the evaluation of average absolute error (MAE). The $t$-test was used for comparison between groups. $P<0.05$ was the threshold for significance.

\section{Experimental Results}

\subsection{The BP Neural Network Optimized by GA}

3.1.1. The Displacement Measurement. In the experiment of displacement measurement, the temperature in the control room is constant. The end surface of the probe and cellophane is wiped using alcohol to prevent dust and stains from affecting the measurement results. The angle between the probe and the target substance is $90^{\circ}$. Before the measurement, the laser is preheated. The probe fits closely to the aluminum workpiece surface, and the movement distance is controlled at $0.05 \mathrm{~mm}$ each time. The upper position machine is used to read the power value of the channels $\mathrm{CH} 1$ and $\mathrm{CH} 2$, and a curve is drawn to show the relationship between the displacement and the received power (Figure 6).

3.1.2. Comparative Analysis of Optimized GA-BP and Traditional BP Approach in Terms of Accuracy and Error Value. In the parameters of the BP neural network, 2 means input and 1 means output. 7 hidden layer nodes are set, and the target error is 0.0001 . The number of training is 1500 , and the training function is Trainingfu. The population size is set as 100 by GA, with a crossover probability of 0.2 and a mutation probability of 0.05 . After setting of parameters, the power value and displacement value are used as the input and output of the neural network for repeated training, respectively. At the same time, the relevant parameters of the network are adjusted to make the training result reach the predicted value. To test the data, the electric displacement stage moves at $0.1 \mathrm{~mm} / \mathrm{step}$. The power meter is used

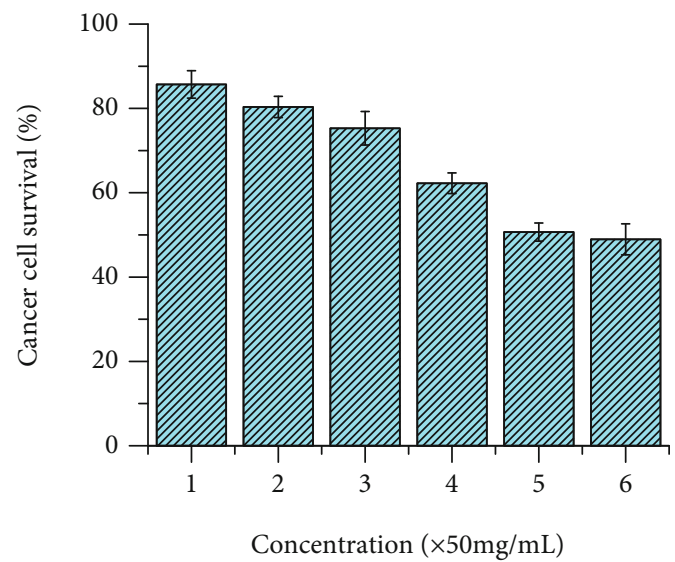

Figure 14: The relationship between cell survival rate and the concentration of $\mathrm{HA}-\mathrm{SiO}_{2} /$ FITC-APTES-NPs.

to obtain the predicted output value and the predicted output value of the BP neural network optimized by GA.

Further, for evaluating the accuracy rates of traditional and optimized GA-BP-based approach, the accuracy parameters have been evaluated for the testing displacements.

From the graphical representation in Figure 7 , it is depicted that the accuracy value increases with a small change in testing displacement for both the traditional and optimized cases. However, it should be noted that the value of accuracy for the optimized GA-BP neural network is much better compared to the traditional BP neural network-based approach. The final accuracy value obtained for the GA-BP method is $94.54 \%$ which is superior to the final accuracy value of $86.78 \%$ obtained for the traditional BP neural network method.

For the comparison of the traditional BP neural network and the GA optimized BP neural network, the RMS and MAE error values are introduced. It is found that the RMS and MAE of the optimized BP neural network are smaller, as shown in Figure 8.

Figure 8 depicts the error value with respect to testing displacement in $(\mathrm{mm})$ for the normal BP predicted value and the true value. It also depicts the error rate between the GA-BP predicted values and the true values for the 


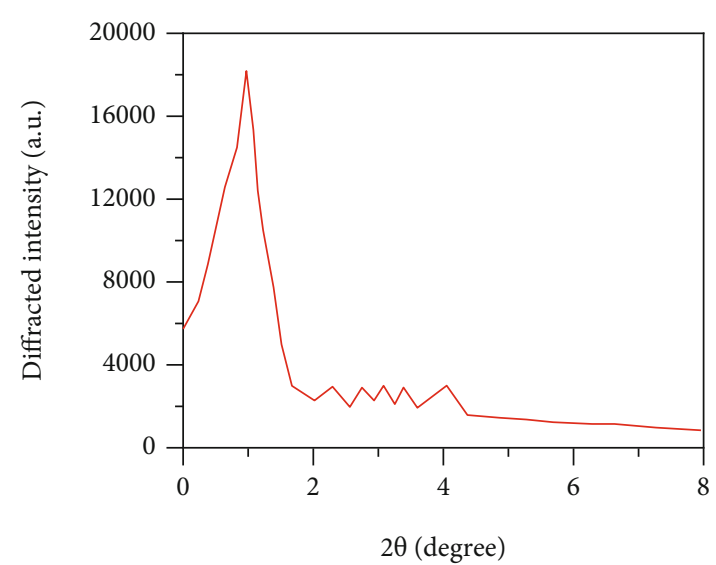

(a)

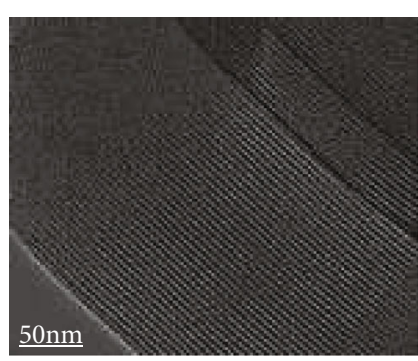

(b)

FIGURE 15: Characterization of CMK-2. Note: (a) diffraction image and (b) EMS.

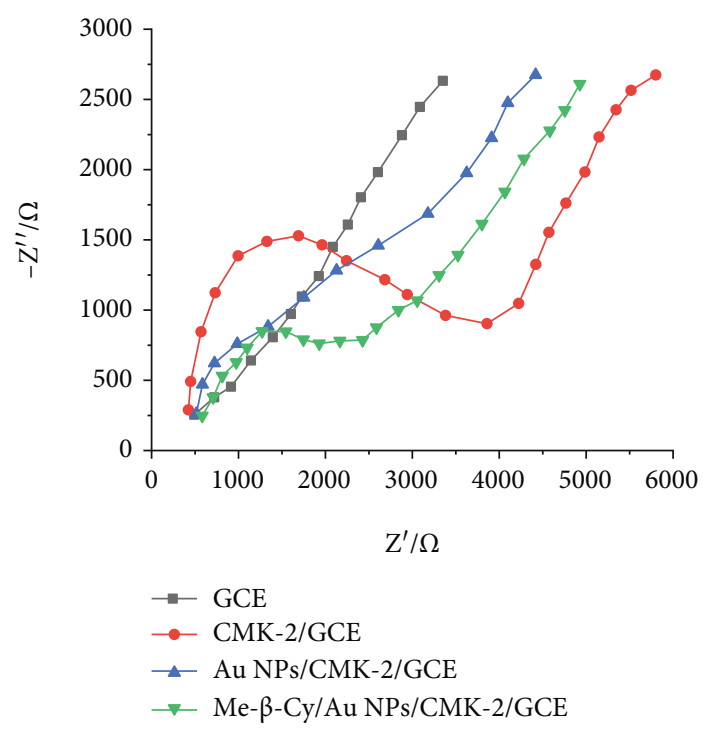

(a)

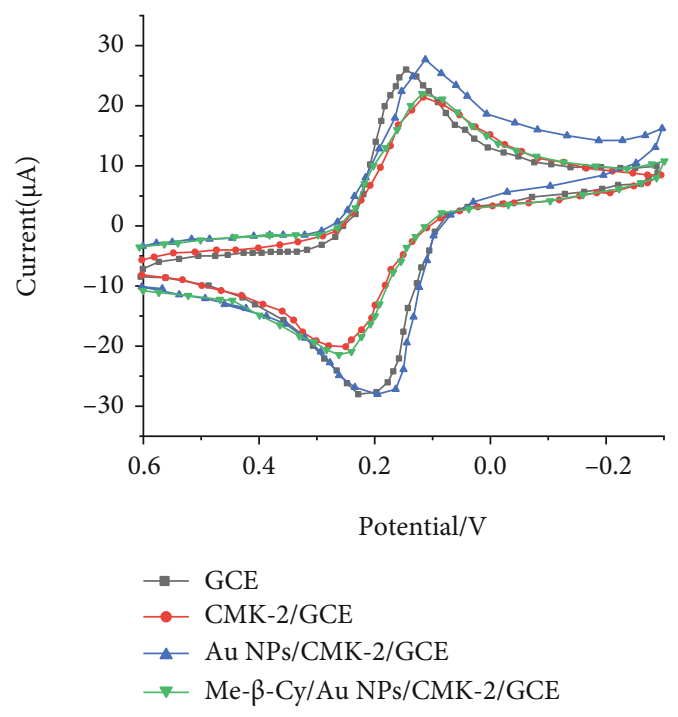

(b)

FIgURE 16: The electrochemical properties of the modified electrodes. (a) Electrochemical impedance curves. (b) CA spectra.

corresponding testing displacement. It can be seen that the error value is minimized near 0 for the case of the GA-BPbased approach which is not minimized much while utilizing the BP predictions alone. Also, it can be noted that the variations in the error values are minimized while utilizing the GA-based optimized BP neural network approach, as compared to the tradition BP neural network-based method.

\subsection{Characterization of Silicon-Coated Fluorescent NPs}

3.2.1. Morphology and Particle Size. It is evident from Figure 9(a) that the silicon-coated fluorescent NPs have good morphology, good dispersion, and relatively uniform particle size, which are sphere in shape. It is evident from Figure 9(b) that the particle size of NPs is about $105 \mathrm{~nm}$.

3.2.2. The Infrared Spectrum. The infrared spectrum is obtained for measurement of FITC, FITC-APTES precursors, $\mathrm{H}_{2} \mathrm{~N}-\mathrm{SiO}_{2} /$ FITC-APTES-NPs, and $\mathrm{HA}_{-} \mathrm{SiO}_{2} /$ FITC-
APTES-NPs. It is evident form Figure 10(a) that the FITC has the characteristic peak of the isothiocyano group at $2186 \mathrm{~cm}^{-1}$ where the FITC-APTES precursor does not possess the characteristic peak. It further indicates that a coupling reaction occurs between FITC and APTES, the isothiocyano group is combined with the guanidine group, and the FITC-APTES precursor is successfully prepared.

It is evident from Figure 10 (b) that $\mathrm{H}_{2} \mathrm{~N}-\mathrm{SiO}_{2} / \mathrm{FITC}$ APTES-NPs have the characteristic peak of amino group appears at $3433 \mathrm{~cm}^{-1}$. The characteristic peak of silica at $1100 \mathrm{~cm}^{-1}$ shows that the prepared NPs contain amino groups. The HA-SiO 2 /FITC-APTES-NPs have the stretching vibration peak of $\mathrm{NH}$ at $3058 \mathrm{~cm}^{-1}$. The stretching vibration peak of $\mathrm{CH}$ is at $2990 \mathrm{~cm}^{-1}$, and characteristic peaks of carboxyl groups are at $1711 \mathrm{~cm}^{-1}$ and $1736 \mathrm{~cm}^{-1}$. These peaks are all caused by the group's stretching vibration, indicating that HA successfully binds to the surface of fluorescent NPs through amide bonds. 
3.2.3. The Fluorescence Property. It is evident from Figure 11(a) that after FITC reacts with APTES to form the FITC-APTES precursor, the overall fluorescence intensity decreases. Though the fluorescence intensity of $\mathrm{H}_{2} \mathrm{~N}$ $\mathrm{SiO}_{2} /$ FITC-APTES-NPs and HA-SiO $/$ /FITC-APTES-NPs decreases slightly, the peak value is still comparatively high. This indicates that the $\mathrm{H}_{2} \mathrm{~N}-\mathrm{SiO}_{2}$ /FITC-APTES-NPs and $\mathrm{HA}-\mathrm{SiO}_{2} /$ FITC-APTES-NPs have relatively good fluorescence properties. The fluorescence confocal microscopic image of $\mathrm{HA}-\mathrm{SiO}_{2}$ /FITC-APTES-NPs shows that the fluorescence NPs are uniformly distributed (Figure 11(b)).

3.2.4. The Chemical Stability. Whether the formation of FITC-APTES will cause the leakage of fluorescent dyes is explored. The $\mathrm{HA}-\mathrm{SiO}_{2} /$ FITC-APTES-NPs are immersed in ultrapure water for $80 \mathrm{~h}$ and compared with the $\mathrm{HA}-\mathrm{SiO}_{2} /$ FITC-NPs for fluorescence intensity. It is found that the fluorescence intensity of $\mathrm{HA}_{-} \mathrm{SiO}_{2} / \mathrm{FITC}-\mathrm{NPs}$ decreases obviously in the first 10 hours, indicating that the dye leakage is serious. The curve gradually becomes stable after 10 hours. The fluorescence intensity of $\mathrm{HA}-\mathrm{SiO}_{2} / \mathrm{FITC}-$ APTES-NPs decreases slowly. The relative fluorescence intensity of $\mathrm{HA}^{-\mathrm{SiO}_{2}}$ /FITC-APTES-NPs in each time period higher than that of $\mathrm{HA}-\mathrm{SiO}_{2} / \mathrm{FITC}-\mathrm{NPs}$ is maintained over $80 \%$ (Figure 12). It shows that the $\mathrm{HA}^{-\mathrm{SiO}_{2} / \text { FITC-APTES- }}$ NPs have good fluorescence stability.

3.2.5. Detection of Hela Tumor Cells. During diagnosis of tumor diseases, specific markers are often used as auxiliary means. HA can specifically recognize the unique HA adhesion factor protein receptor on the surface of cancer cells and binds to the surface of cancer cells. In this study, the HA-modified fluorescent NPs were visualized using a laser confocal microscope (Figure 13). The left was a bright-field optical image, and the right was a dark-field fluorescent image. The cells in the two corresponded to each other. In the fluorescence bright-field image, there was obvious green fluorescence on the surface of cancer cells, which indicated that HA-modified fluorescent NPs covered the surface of cancer cells (Figure 13(a)). In the dark-field image, the cells emitted bright green fluorescence (Figure 13(b)). Therefore, the HA-modified fluorescent NPs are a new method for early diagnosis of cancer cells.

3.2.6. Cell Survival Rate. In this study, the MTT method was used to determine the survival rate of cells. After MTT treatment of living cells, the mitochondrial-specific dehydrogenase in the cell would cause MTT to form water-insoluble blue-purple crystal formazan, which would not be formed in dead cells. To measure the absorbance of different concentrations of HA-modified fluorescent NPs at $580 \mathrm{~nm}$, cells cultured in DMEM were set as blank group, and the cells cultured with fluorescent NPs were set as the control group. A lower survival rate indicates greater toxicity of HAmodified fluorescent NPs to cancer cells. It was found that as the concentration increased, the survival rate of cancer cells was gradually decreasing (Figure 14). To maintain good fluorescence intensity and cell survival rate, $50 \mathrm{mg} / \mathrm{mL}$ fluorescent NPs were selected for cell incubation experiments.

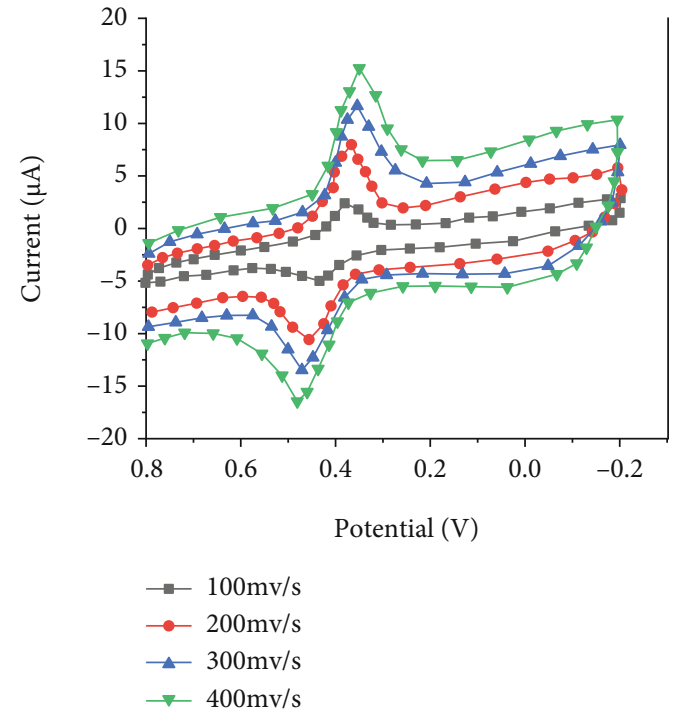

Figure 17: The CV spectra of Me- $\beta$-Cy/Au NPs/CMK-2/GCE at different scanning speeds.

\subsection{The Characterization of the Modified Electrode}

3.3.1. Characterization of CMK-2. The Rigaku Corporation SA-HF3 (Rigaku Corporation, Japan) is used to obtain the $\mathrm{X}$-ray diffraction image of CMK-2. It is found that there is an obvious diffraction peak that appears at $2 \theta$, indicating that CMK-2 has an obvious two-dimensional hexagonal structure (Figure 15(a)). The JEOL-2100 instrument (JEOL Company, Japan) is used to obtain the transmission EMS image of CMK-2. It is noted that it has a clear stripe structure and uniform size, indicating that the CMK-2 has a relatively good stripe structure (Figure 15(b)).

3.3.2. Electrochemical Properties of Modified Electrodes. Electrochemical impedance spectroscopy (EIS) can be used for the characterization of different electrodes. In this study, the impedance change during the modification of the electrode is explored, and further, the electrochemical impedance spectroscopy is observed. The electrochemical impedance of bare glass carbon electrode (GCE) CMK-2/GCE, Au NPs/ CMK-2/GCE, and Me- $\beta$-Cy/Au NPs/CMK-2/GCE was measured in $1 \mathrm{mmol} / \mathrm{L} \quad \mathrm{Fe}(\mathrm{CN})_{6}^{3-/ 4-}$ phosphate buffer (Figure 16(a)). The frequency change range is $0.01-10 \mathrm{kHz}$.

The electron transfer resistance of bare GCE is very small, about $500 \Omega$. After it is modified by CMK-2, the electron transfer resistance rapidly increases to $3800 \Omega$. The reason may be that the mesoporous silicon material itself does not conduct electricity. After electrodeposition $\mathrm{Au}$, the impedance value is reduced, which shows that the Au NPs has good conductivity and promotes the transfer of $\mathrm{Fe}(\mathrm{CN})_{6}{ }^{3-14-}$. After it is combined with $\mathrm{Me}-\beta-\mathrm{Cy}$, the electron transfer resistance continues to increase to $2100 \Omega$. It may be that the organic matter Me- $\beta$-Cy inhibits the transfer of $\mathrm{Fe}(\mathrm{CN})_{6}{ }^{3-14-}$. Taken together, it can be confirmed that $\mathrm{Me}-\beta$-Cy/Au NPs/CMK-2/GCE is successfully prepared in the study. Figure 16(b) shows the CV spectra of bare GCE, CMK-2/GCE, Au NPs/CMK-2/GCE, and Me- $\beta$-Cy/Au 


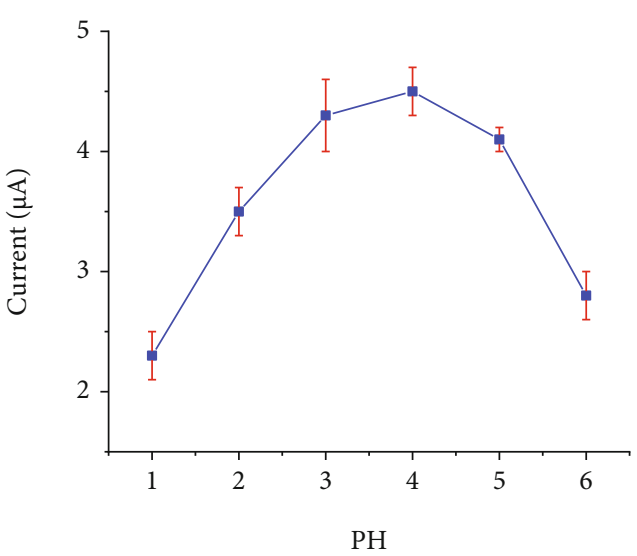

(a)

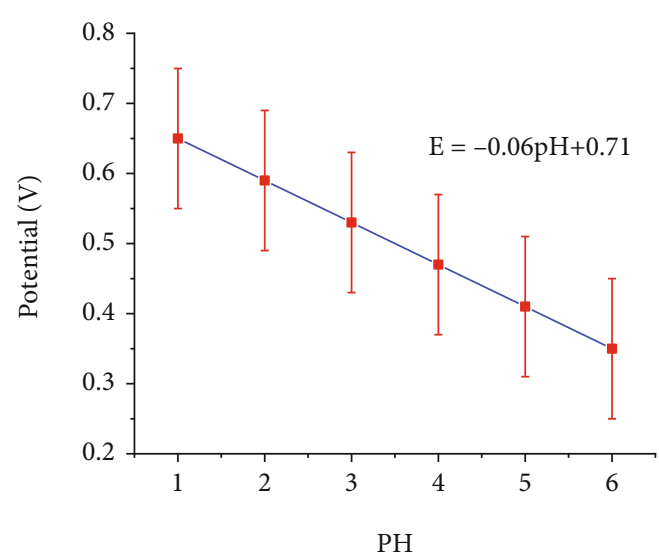

(b)

FIGURE 18: The influence of the $\mathrm{pH}$ value on modified electrode. Note: (a) the relationship curve between the peak current value and pH and (b) the linear correlation curve between reduction peak potential and $\mathrm{pH}$ of modified electrode.

$\mathrm{NPs} / \mathrm{CMK}-2 / \mathrm{GCE}$ in $1 \mathrm{mmol} / \mathrm{L} \mathrm{Fe}(\mathrm{CN})_{6}^{3-/ 4-}$ phosphate buffer solution. The impedance changes in Figure 16(a) corresponded to that in Figure 16(b).

3.3.3. The Peak Current and Scanning Speed. Figure 17 shows the CA spectra of Me- $\beta$-Cy/Au NPs/CMK-2/GCE in a phosphate buffer at different scanning speeds. The scanning speed is controlled at $100-400 \mathrm{mv} / \mathrm{s}$, and the results show that the scanning speed continues to increase providing the current peak value highest at $0.35 \mathrm{~V}$.

3.3.4. The Optimal $p H$ of the Solution. In this study, the influence of the $\mathrm{pH}$ value of the solution on the modified electrode is determined along with the relationship curve between the peak current value and $\mathrm{pH}$ drawn. The base solution is $15 \mu \mathrm{mol} / \mathrm{L}$ phosphate buffer with different $\mathrm{pH}$ levels. It is noted that as the $\mathrm{pH}$ value in the solution gradually increases from 1.0 to 4.0 , the peak current signal also increases. When the $\mathrm{pH}$ value exceeds 4.0 , the response signal decreases rapidly (Figure 18(a)). It may be that when the acidity of the solution increases, the number of protons is gradually decreasing, affecting the reaction on the electrode. When the $\mathrm{pH}$ is controlled in the range of 1.0-6.0, the reduction peak potential has a linear relationship with the $\mathrm{pH}$ value of the test solution. The slope of the fitting line is $-60 \mathrm{mV}$, which is very close to the theoretical value $-59 \mathrm{mV}$. It suggests that during the electrochemical reaction, the number of protons is equal to that of electrons (Figure 18(b)). When $\mathrm{pH}=4.0$, the peak current is the highest. Hence, $\mathrm{pH}=4.0$ was selected as the best $\mathrm{pH}$ value.

3.3.5. The Time for Modification. Me- $\beta$-Cy bonds to the gold NPs through S-Au bond, but this kind of covalent bond takes a certain time to achieve a stable state. Therefore, the time needed to reach a stable state is explored during Me$\beta$-Cy modification. The reduction peak current in $15 \mu \mathrm{mol} /$ $\mathrm{L}$ phosphate buffer is recorded with time, as shown in Figure 19.

The results show that when the modification time of Me$\beta$-Cy is less than $15 \mathrm{~h}$, the reduction peak current gradually

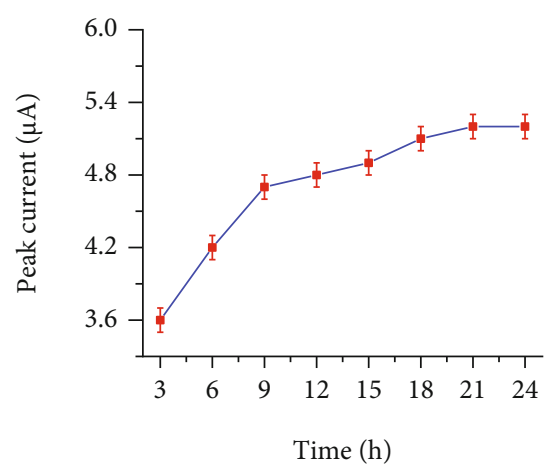

FIgURE 19: The reduction peak current with time.

increases with the time. After $15 \mathrm{~h}$, the peak current tends to be stable. In this study, the modification time is set as $15 \mathrm{~h}$.

\section{Conclusion}

In the study, the GA is used to optimize the BP neural network, and the identification NP sensor is used to diagnose cancer cells, aimed at realizing the early diagnosis of cancer cells by fluorescence imaging. The RMS and MAE of the optimized BP neural network are analyzed which were observed to be smaller than the traditional one. Furthermore, the modified electrode Me- $\beta$-Cy/Au NPs/CMK-2/ GCE is successfully prepared and applied to the sensor. This sensor shows good stability, selectivity, and anti-interference property, providing a new method for the detection of early cancer cells. The prepared HA-modified fluorescent NPs have good fluorescence stability, and HA covalently modified fluorescent NPs can specifically bind to cancer cells. This method can be used in fluorescence imaging detection. This study provides a good theoretical basis for clinical diagnosis and prevention of cancer. However, the practical implementation of results using cervical cancer cells are universally applicable to other tumor cells, and its further verification will be the future research directions of this research work. 


\section{Data Availability}

All data has been provided in the main manuscript.

\section{Conflicts of Interest}

The authors declare that they have no conflicts of interest.

\section{References}

[1] H. K. Jia, L. D. Yu, Y. Z. Jiang, H. N. Zhao, and J. M. Cao, "Compensation of rotary encoders using Fourier expansionback propagation neural network optimized by genetic algorithm," Sensors (Basel), vol. 20, no. 9, p. 2603, 2020.

[2] J. He, C. Pan, Y. Liu, and X. du, "Quantitative analysis of carbon with laser-induced breakdown spectroscopy (LIBS) using genetic algorithm and back propagation neural network models," Applied Spectroscopy, vol. 73, no. 6, pp. 678-686, 2019.

[3] J. Luo, R. Ren, and K. Guo, “The deformation monitoring of foundation pit by back propagation neural network and genetic algorithm and its application in geotechnical engineering," PLoS One, vol. 15, no. 7, article e0233398, 2020.

[4] Y. X. Hou, H. F. Zhao, Z. Zhang, and K. N. Wu, "A novel method for predicting cadmium concentration in rice grain using genetic algorithm and back-propagation neural network based on soil properties," Environmental Science and Pollution Research International, vol. 25, no. 35, pp. 35682-35692, 2018.

[5] Q. Li, H. Tao, J. Wang et al., "Warfarin maintenance dose prediction for patients undergoing heart valve replacement- a hybrid model with genetic algorithm and back-propagation neural network," Scientific Reports, vol. 8, no. 1, p. 9712, 2018.

[6] C. Huang, X. Jia, and Z. Zhang, "A modified back propagation artificial neural network model based on genetic algorithm to predict the flow behavior of 5754 aluminum alloy," Materials (Basel), vol. 11, no. 5, p. 855, 2018.

[7] J. Shen, Y. Cui, J. Gu, Y. Li, and L. Li, "A genetic algorithmback propagation artificial neural network model to quantify the affinity of flavonoids toward P-glycoprotein," Combinatorial Chemistry \& High Throughput Screening, vol. 17, no. 2, pp. 162-172, 2014.

[8] P. He, Y. Fan, B. Pan, Y. Zhu, J. Liu, and D. Zhu, "Calibration and verification of dynamic particle flow parameters by the back-propagation neural network based on the genetic algorithm: recycled polyurethane powder," Materials (Basel), vol. 12, no. 20, p. 3350, 2019.

[9] K. Shiba, R. Tamura, T. Sugiyama et al., "Functional nanoparticles-coated nanomechanical sensor arrays for machine learning-based quantitative odor analysis," ACS Sens, vol. 3, no. 8, pp. 1592-1600, 2018.

[10] M. Waldiya, D. Bhagat, N. R et al., "Development of highly sensitive $\mathrm{H}_{2} \mathrm{O}_{2}$ redox sensor from electrodeposited tellurium nanoparticles using ionic liquid," Biosensors \& Bioelectronics, vol. 132, pp. 319-325, 2019.

[11] S. Wang, R. Farnood, and N. Yan, "Corn-derived dendrimerlike carbohydrate phytoglycogen nanoparticles as selective fluorescent sensor for silver ions," Carbohydrate Polymers, vol. 223, p. 115095, 2019.

[12] Y. T. Yaman, O. Akbal, and S. Abaci, "Development of clayprotein based composite nanoparticles modified single-used sensor platform for electrochemical cytosensing application," Biosensors \& Bioelectronics, vol. 132, pp. 230-237, 2019.
[13] Y. Saylan, F. Yılmaz, A. Derazshamshir, E. Yılmaz, and A. Denizli, "Synthesis of hydrophobic nanoparticles for realtime lysozyme detection using surface plasmon resonance sensor," Journal of Molecular Recognition, vol. 30, no. 9, 2017.

[14] M. M. Rahman, J. Ahmed, and A. M. Asiri, "Thiourea sensor development based on hydrothermally prepared CMO nanoparticles for environmental safety," Biosensors \& Bioelectronics, vol. 99, pp. 586-592, 2018.

[15] F. A. Ozdemir Olgun, A. Üzer, B. D. Ozturk, and R. Apak, “A novel cerium oxide nanoparticles-based colorimetric sensor using tetramethyl benzidine reagent for antioxidant activity assay," Talanta, vol. 182, pp. 55-61, 2018.

[16] H. Lu and S. Xu, "Visualizing BPA by molecularly imprinted ratiometric fluorescence sensor based on dual emission nanoparticles," Biosensors \& Bioelectronics, vol. 92, pp. 147-153, 2017.

[17] Z. Khosroshahi, F. Karimzadeh, M. Kharaziha, and A. Allafchian, "A non-enzymatic sensor based on threedimensional graphene foam decorated with $\mathrm{Cu}-\mathrm{xCu}_{2} \mathrm{O}$ nanoparticles for electrochemical detection of glucose and its application in human serum," Materials Science \& Engineering. C, Materials for Biological Applications, vol. 108, p. 110216, 2020.

[18] Z. Yang, D. Y. Wang, Y. Pang et al., "Simultaneously detecting subtle and intensive human motions based on a silver nanoparticles bridged graphene strain sensor," ACS Applied Materials \& Interfaces, vol. 10, no. 4, pp. 3948-3954, 2018.

[19] D. Li, S. Yu, C. Sun, C. Zou, H. Yu, and K. Xu, "U-shaped fiberoptic ATR sensor enhanced by silver nanoparticles for continuous glucose monitoring," Biosensors \& Bioelectronics, vol. 72, pp. 370-375, 2015.

[20] J. S. Lee, B. Peng, A. C. Sabuncu et al., "Multiple consecutive recapture of rigid nanoparticles using a solid-state nanopore sensor," Electrophoresis, vol. 39, no. 5-6, pp. 833-843, 2018.

[21] F. Amiripour, S. N. Azizi, and S. Ghasemi, "Gold-copper bimetallic nanoparticles supported on nano P zeolite modified carbon paste electrode as an efficient electrocatalyst and sensitive sensor for determination of hydrazine," Biosensors \& Bioelectronics, vol. 107, pp. 111-117, 2018.

[22] H. Wang, Y. Xie, S. Liu et al., "Presence of fluorescent carbon nanoparticles in baked lamb: their properties and potential application for sensors," Journal of Agricultural and Food Chemistry, vol. 65, no. 34, pp. 7553-7559, 2017.

[23] S. E. Jin, J. E. Jin, W. Hwang, and S. W. Hong, "Photocatalytic antibacterial application of zinc oxide nanoparticles and selfassembled networks under dual UV irradiation for enhanced disinfection," International Journal of Nanomedicine, vol. Volume 14, pp. 1737-1751, 2019.

[24] S. Lotfi and H. Veisi, "Pd nanoparticles decorated [email protected $] / \mathrm{Fe}_{3} \mathrm{O}_{4}$ nanocomposite modified glassy carbon electrode as a new electrochemical sensor for simultaneous determination of acetaminophen and phenylephrine," Materials Science \& Engineering. C, Materials for Biological Applications, vol. 105, p. 110112, 2019.

[25] D. Refaat, M. G. Aggour, A. A. Farghali et al., "Strategies for molecular imprinting and the evolution of MIP nanoparticles as plastic antibodies-synthesis and applications," International Journal of Molecular Sciences, vol. 20, no. 24, p. 6304, 2019.

[26] X. Jin, G. Fang, M. Pan, Y. Yang, X. Bai, and S. Wang, "A molecularly imprinted electrochemiluminescence sensor based on upconversion nanoparticles enhanced by electrodeposited rGO for selective and ultrasensitive detection of 
clenbuterol," Biosensors \& Bioelectronics, vol. 102, pp. 357364, 2018.

[27] Z. Q. Zheng, J. D. Yao, B. Wang, and G. W. Yang, "Light-controlling, flexible and transparent ethanol gas sensor based on $\mathrm{ZnO}$ nanoparticles for wearable devices," Scientific Reports, vol. 5, no. 1, 2015.

[28] H. Shibata, Y. Ikeda, and D. Citterio, "Inkjet-printed colorimetric paper-based gas sensor arrays for the discrimination of volatile primary amines with amine-responsive dyeencapsulating polymer nanoparticles," Methods in Molecular Biology, vol. 2027, pp. 101-114, 2019. 